patient-used flexible endoscopes before and after cleaning. Am J Infect Control 1999;27(5):392-401.

10. Roberts CG. Studies on the bioburden on medical devices and the importance of cleaning. In: Rutala WA, ed. Disinfection, Sterilization and Antisepsis: Principles and Practices in Healthcare Facilities. Washington, DC: Association for Professionals in Infection Control and Epidemiology, 2001:63-69.

11. Rutala WA, Gergen MF, Weber DJ. Efficacy of a washer-disinfector in eliminating healthcare-associated pathogens from surgical instruments. Infect Control Hosp Epidemiol 2014;35(7):883885 .

\section{Room Decontamination Using an Ultraviolet-C Device with Short Ultraviolet Exposure Time}

Disinfection of noncritical room surfaces and equipment is normally performed by manually applying a liquid disinfectant with a cloth, wipe, or mop. Studies have shown 10\%$50 \%$ of the surfaces in rooms with patients colonized or infected with Clostridium difficile, methicillin-resistant Staphylococcus aureus (MRSA), and vancomycin-resistant Enterococcus (VRE) are contaminated with these pathogens, and a lack of thoroughness in cleaning contaminated surfaces in such rooms (mean, $32 \%$ of surfaces and objects cleaned) has been linked to a $120 \%$ increase in risk of infection to the next occupant in that room. ${ }^{1,2}$ These data have led to efforts to improve surface disinfection practices and the development of "no-touch" room decontamination units that avoid the problems associated with manual disinfection. ${ }^{3}$

Room decontamination units that use ultraviolet-C (UVC, $254 \mathrm{~nm}$ ) are commercially available and have been shown to effectively decontaminate surfaces in patient rooms. ${ }^{4-8}$ The purpose of this study was to determine whether a fixed cycletime UV-C device was effective in inactivating the test bacteria in a patient room with and without reflective coating. To better understand the effect of the reflective coating, the UV-
C intensity on surfaces of interest were directly measured using a UV-C radiometric sensor.

We investigated a single, easily transportable UV-C device (V-360+, UltraViolet Devices) that incorporates four 64-inch UV-C lamps having a total output of 1,200 W. The device's cycle time was determined by the manufacturer on the basis of the size and configuration of various-sized rooms. Measurements were performed in 2 patient rooms as described previously. $^{8}$

Testing was performed as previously reported using C. difficile spores and a clinical isolate of MRSA. ${ }^{8}$ The room decontamination times were fixed at 5 minutes for MRSA and 10 minutes for $C$. difficile spores. Following cycle completion, each Formica template was cultured, and after incubation, the colony-forming units of the test organisms on each plate were quantified.

Measurements of UV-C irradiance energy $\left(\mathrm{W} / \mathrm{cm}^{2}\right)$ were performed using a radiometer (ILT1700 Research Radiometer, International Light Technologies) equipped with a calibrated, National Institute of Standards and Technology-traceable UV-C detector with appropriate filter and diffuser (SED240/ NS254/W, International Light Technologies).

For disinfection of MRSA with a 5-minute cycle time, we observed a 3.56- $\log _{10}$ reduction without the reflective coating and $4.50-\log _{10}$ reduction with the reflective coating. For disinfection of $C$. difficile spores with a 10-minute cycle time, we observed a $2.78-\log _{10}$ reduction without the reflective coating and 3.05- $\log _{10}$ reduction with the reflective coating (Table 1). The most significant improvements when a reflective wall coating was used were seen on indirect surfaces, where a 1.47$\log _{10}$ reduction increase was observed for MRSA and a 0.81$\log _{10}$ reduction increase was observed for $C$. difficile spores. Measurements of UV-C irradiance were roughly $1 \times 10^{-3} \mathrm{~W} /$ $\mathrm{cm}^{2}$ for direct surfaces in both rooms. For indirect surfaces, however, the reflective coating increased the UV-C irradiance tenfold from $3.7 \times 10^{-6}$ to $4.5 \times 10^{-5}$ (Figure 1).

These results confirm earlier findings that UV-C devices can effectively disinfect patient rooms. This unit achieved a total $3.56-\log _{10}$ reduction (4.10 direct, 2.74 indirect) for MRSA in 5 minutes and a total $2.78-\log _{10}$ reduction $(3.35$

TA в LE 1. Ultraviolet-C Decontamination of Formica Surfaces in Patient Rooms That Were Experimentally Contaminated with Methicillin-Resistant Staphylococcus aureus (MRSA) and Clostridium difficile Spores with and without a Reflective Coating on Walls

\begin{tabular}{lccccc}
\hline & \multicolumn{2}{c}{ MRSA } & & \multicolumn{2}{c}{ C. difficile } \\
\cline { 2 - 3 } \cline { 6 - 7 } Variable & Without coating & With coating & & Without coating & With coating \\
\hline Cycle time, minutes & 5 & 5 & & 10 & 10 \\
Direct surfaces & $4.10(3.88-4.32) ; 30$ & $4.68(4.61-4.76) ; 30$ & & $3.35(3.14-3.55) ; 30$ & $3.34(3.10-3.59) ; 30$ \\
Indirect surfaces & $2.74(2.53-2.94) ; 20$ & $4.21(4.00-4.42) ; 20$ & & $1.80(1.36-2.24) ; 20$ & $2.61(2.24-2.97) ; 20$ \\
$\quad$ Overall & $3.56(3.31-3.80) ; 50$ & $4.50(4.38-4.61) ; 50$ & & $2.78(2.48-3.07) ; 50$ & $3.05(2.82-3.28) ; 50$ \\
\hline
\end{tabular}

Note. Data are mean $\log _{10}$ reduction in colony-forming units (95\% confidence interval) and no. of samples, unless otherwise indicated. Patient room is 130 square feet $\left(12.077 \mathrm{~m}^{2}\right)$ in area. Confidence intervals were calculated based on a Poisson distribution. 


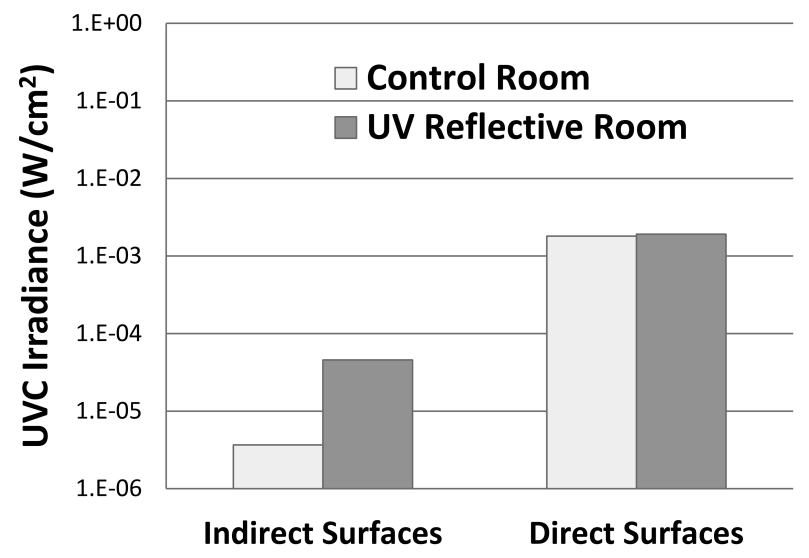

FIGURE 1. Comparison of the average ultraviolet (UV) irradiance $\left(\mathrm{W} / \mathrm{cm}^{2}\right)$ of surfaces directly and indirectly illuminated by the UV$\mathrm{C}$ device.

direct, 1.80 indirect) for $C$. difficile spores in 10 minutes without the reflective coating, compared with a total 3.94- $\log _{10}$ reduction (4.31 direct, 3.85 indirect) for MRSA within 15 minutes and a total $2.79-\log _{10}$ reduction (4.04 direct, 2.43 indirect) for $C$. difficile spores in 50 minutes using another UV-C device for patient rooms without the reflective coating. ${ }^{4}$ Our preliminary data suggest that similar degrees of inactivation may be achieved at a $67 \%$ reduction in exposure time for vegetative bacteria and $80 \%$ reduction in exposure time for $C$. difficile spores without the reflective coating. The differences observed between the UV-C devices may be attributed to the design of the devices (eg, reflector), a factor that should be further investigated.

Furthermore, this study has shown that, for a fixed cycle time, the level of disinfection increases when a UV-C reflective coating has been applied to the walls of the room. Of interest was the amount of improvement for those surfaces not in direct line of sight of the device. One concern of UV-C disinfection relative to other methods, such as hydrogen peroxide vapor, is that it is less effective for non-line-of-sight decontamination. This investigation demonstrated that a UV$\mathrm{C}$ reflective coating can help disperse UV-C to indirect surfaces that are normally shadowed by objects in the room. The use of the reflective coating significantly improved the $\log _{10}$ reduction for direct (MRSA), indirect (MRSA/C. difficile) and total surfaces (MRSA). The reflective coating is $65 \%$ UV reflective at $254 \mathrm{~nm}$ compared with standard paint, which is $3 \%-7 \%$ UV reflective. ${ }^{8}$ Measurements of UV-C irradiance show over a tenfold increase for indirect surfaces, which correlates well with the significant improvement observed in $\log _{10}$ reduction. The fixed cycle times studied should only be used in single-patient rooms in accordance with the manufacturer's directions, because the unit lacks a sensor that adjusts the duration of UV-C delivery on the basis of room size.

In summary, UV-C technology can effectively reduce en- vironmental contamination and should be considered when the environmental mode of transmission is significant (eg, after discharge of patients under contact precautions). This UV-C device tested allows an approximately $3-\log _{10}$ to greater than $4-\log _{10}$ reduction in MRSA on surfaces in 5 minutes and an approximately $2-\log _{10}$ to $3-\log _{10}$ reduction in C. difficile spores on surfaces in 10 minutes without the reflective coating. The use of the reflective coating significantly improved microbial reduction. This allows UV-C disinfection technology to be more easily integrated into healthcare facilities where occupancy is high and fast patient room turnaround time is critical.

\section{ACKNOWLEDGMENTS}

We thank Ultraviolet Devices for loaning University of North Carolina Health Care the ultraviolet-C device used in these experiments and Dr. Emily E. Sickbert-Bennett for statistical assistance.

Financial support. No other financial or technical support was received by University of North Carolina (UNC) Health Care, and Ultraviolet Devices had no involvement in writing or reviewing this article. We acknowledge the use of rooms in the UNC Clinical Translational Research Center (National Institutes of Health grant 1ULRR025747-01).

Potential conflicts of interest. B.M.T. reports that he is the co-inventor of the patent-pending reflective coating and co-owner of Lumacept, which makes the reflective coating. W.A.R. reports that he is a consultant to Advanced Sterilization Products and Clorox. D.J.W. reports that he is a consultant to Clorox and Johnson \& Johnson. All other authors report no conflicts of interest relevant to this article. All authors submitted the ICMJE Form for Disclosure of Potential Conflicts of Interest, and the conflicts that the editors consider relevant to this article are disclosed here.

\section{William A. Rutala, PhD, MPH; ${ }^{1,2}$ Maria F. Gergen, MT (ASCP); ${ }^{1}$ Brian M. Tande, $\mathrm{PhD} ;{ }^{3}$ David J. Weber, MD, MPH ${ }^{1,2}$}

Affiliations: 1. Hospital Epidemiology, University of North Carolina Health Care, Chapel Hill, North Carolina; 2. Division of Infectious Diseases, University of North Carolina School of Medicine, Chapel Hill, North Carolina; 3. Department of Chemical Engineering, University of North Dakota, Grand Forks, North Dakota.

Address correspondence to William Rutala, PhD, MPH, 130 Mason Farm Road, Bioinformatics, Chapel Hill, NC 27599 (brutala@unch.unc.edu).

Received January 14, 2014; accepted March 15, 2014; electronically published June 30, 2014.

Infect Control Hosp Epidemiol 2014;35(8):1070-1072

(C) 2014 by The Society for Healthcare Epidemiology of America. All rights reserved. 0899-823X/2014/3508-0023\$15.00. DOI: 10.1086/677149

\section{REF E R E N C ES}

1. Otter JA, Yezli S, Salkeld JAG, French GL. Evidence that contaminated surfaces contribute to the transmission of hospital pathogens and an overview of strategies to address contaminated surfaces in hospital settings. Am J Infect Control 2013;41:S6-S11.

2. Carling PC. Methods for assessing the adequacy of practice and improving room disinfection. Am J Infect Control 2013;41:S20S25. 
3. Rutala WA, Weber DJ. Disinfectants used for environmental disinfection and new room decontamination technology. Am J Infect Control 2013;41:S36-S41.

4. Rutala WA, Gergen MF, Weber DJ. Room decontamination by ultraviolet radiation. Infect Control Hosp Epidemiol 2010;31:10251029.

5. Anderson DJ, Gergen MF, Smathers E, et al. Decontamination of targeted pathogens from patient rooms using an automated ultraviolet-C-emitting device. Infect Control Hosp Epidemiol 2013; 34:465-471.

6. Boyce JM, Havill NL, Moore BA. Terminal decontamination of patient rooms using an automated mobile UV light unit. Infect Control Hosp Epidemiol 2011;32:743-747.

7. Nerandzic MM, Cadnum JL, Pultz MJ, Donskey CJ. Evaluation of an automated ultraviolet radiation device for decontamination of Clostridium difficile and other healthcare-associated pathogens in hospital rooms. BMC Infect Dis 2010;10:197.

8. Rutala WA, Gergen MF, Tande BM, Weber DJ. Rapid hospital room decontamination using ultraviolet (UV) light with a nanostructured UV-reflective wall coating. Infect Control Hosp Epidemiol 2013;34:527-529.

\section{Brucella abortus Exposure during an Orthopedic Surgical Procedure in New Mexico, 2010}

Brucellosis, a zoonotic disease that can be transmitted through inhalation of infectious aerosolized particles, is endemic in many areas, including Mexico. ${ }^{1-4}$ Manifestations of disease can range from subclinical illness to osteoarticular disease and chronic sequelae. ${ }^{4}$ It is a potential occupational hazard among laboratory workers. ${ }^{3}$ Although Brucella infection is not usually a risk to medical staff, prosthetic joint infections have been encountered during surgery. ${ }^{5-9}$ We report a case of periprosthetic Brucella infection and the subsequent investigation into possible transmission to operating room and laboratory staff. Objectives of the investigation included infection prevention, case-finding, and examination into potential routes of Brucella species transmission.

The New Mexico Department of Health (NMDOH), in consultation with the Centers for Disease Control and Prevention (CDC), initiated an investigation of operating room and laboratory staff exposures. Among operating room staff, high-risk exposures were defined as presence in the operating room during aerosol-generating procedures, including joint irrigation and cleaning after the procedure. $\mathrm{NMDOH}$ Scientific Laboratory Division and reference laboratory staff involved in testing the patient's isolate were contacted to evaluate laboratory exposures. Serial serologic testing and antibiotic postexposure prophylaxis (PEP; $100 \mathrm{mg}$ doxycycline orally twice daily and rifampin $600 \mathrm{mg}$ once daily for 21 days, for those without contraindications) was recommended for individuals with high-risk exposures. ${ }^{10}$ The CDC performed serologic testing for anti-Brucella antibodies by microagglutination.

The 67-year-old female patient was born in, raised in, and frequently traveled to Mexico. Her first hip replacement occurred in Mexico 2 years before presentation for revision. During revision, implant component loosening, bone loss, and cloudy synovial fluid were noted. Synovial fluid was cultured, the joint was debrided and copiously irrigated, and hip replacement was deferred; an articulating vancomycinand tobramycin-impregnated cement spacer was placed. Growth suggestive of Brucella species resulted from synovial fluid culture at a reference laboratory. The NMDOH Scientific Laboratory Division conducted confirmatory nucleic acid amplification testing, and subsequently the CDC performed speciation; Brucella abortus was identified.

Seventeen high-risk exposures and 1 low-risk exposure were investigated; fifteen high-risk exposures occurred in the operating room. Personal protective equipment (PPE) varied from body exhaust suits (surgeon, first assistant, and scrub technician) to gloves only (cleaning staff); none wore N95 respiratory protection. Because the joint was copiously irrigated, hospital staff who cleaned the operating room were also considered to be exposed. One low- and 2 high-risk reference laboratory staff exposures occurred during isolate processing outside of the biosafety cabinet on an open bench; the low-risk exposure occurred outside the 5 -foot $(1.5-\mathrm{m})$ radius for exposures that qualified as high risk. ${ }^{10}$ No exposures occurred at the NMDOH Scientific Laboratory Division, because the isolate was handled inside a biosafety cabinet.

Fifteen exposed operating room staff underwent serial serologic testing and prophylaxis. Reference laboratory employees with high-risk exposures agreed to serologic testing but declined PEP. All who elected prophylaxis completed the PEP regimen. None of those exposed met criteria for seroconversion (ie, fourfold increase in anti-Brucella antibody titer). Two individuals whose total antibody titers were indeterminate (between $1: 20$ and $1: 40$, potentially resulting from test run variation and assay cross-reaction with other antibodies) were referred for infectious disease consultation; no evidence of acute Brucella infection was detected. Exposed individuals self-monitored and were observed by personal healthcare or occupational medicine providers for 6 months; none developed symptoms of brucellosis.

The surgical patient was treated for 3 months with combination therapy (doxycycline and rifampin) to address osteomyelitis and prevent Brucella infection relapse. A preoperative aspirate, before reimplantation of the hip replacement, yielded a negative culture result. The $\mathrm{NMDOH}$ recommended that anyone involved in reimplantation use N95 masks and goggles, minimize aerosol-generating procedures, and handle biological specimens with care. The patient's recovery was uneventful without evidence of infection recurrence at 2 years of follow-up.

This case report demonstrates the need to consider evaluation for Brucella species infection and risk factors among 